\title{
Anatomy of Zero-norm States in String Theory
}

\author{
Chuan-Tsung Chan* \\ Physics Division, National Center for Theoretical Sciences, Hsinchu, Taiwan, R.O.C. \\ Jen-Chi Lee ${ }^{\dagger}$ and Yi-Yang $\ddagger$ \\ Department of Electrophysics, National Chiao-Tung University, Hsinchu, Taiwan, R.O.C.
}

(Dated: September 29, 2018)

\begin{abstract}
We calculate and identify the counterparts of zero-norm states in the old covariant first quantised (OCFQ) spectrum of open bosonic string in two other quantization schemes of string theory, namely the light-cone DDF zero-norm states and the off-shell BRST zero-norm states (with ghost) in the Witten string field theory (WSFT). In particular, special attention is paid to the inter-particle zero-norm states in all quantization schemes. For the case of the off-shell BRST zero-norm states, we impose the no ghost conditions and recover exactly two types of on-shell zero-norm states in the OCFQ string spectrum for the first few low-lying mass levels. We then show that off-shell gauge transformations of WSFT are identical to the on-shell stringy gauge symmetries generated by two types of zero-norm states in the generalized massive $\sigma$-model approach of string theory. The high energy limit of these stringy gauge symmetries was recently used to calculate the proportionality constants, conjectured by Gross, among high energy scattering amplitudes of different string states. Based on these zero-norm state calculations, we have thus related gauge symmetry of WSFT to the high-energy stringy symmetry of Gross.
\end{abstract}

\footnotetext{
*Electronic address: ctchan@phys.cts.nthu.edu.tw

$\dagger$ Electronic address: jcclee@cc.nctu.edu.tw

${ }^{\ddagger}$ Electronic address: yiyang@mail.nctu.edu.tw
} 


\section{INTRODUCTION}

Recently it was discovered that $[1,2]$ the high-energy limit $\alpha^{\prime} \rightarrow \infty$ of stringy Ward identities, derived from the decoupling of two types of zero-norm states in the OCFQ spectrum, imply an infinite number of linear relations among high energy scattering amplitudes of different string states with the same momenta. Moreover, these linear relations can be used to fix the proportionality constants between high energy scattering amplitudes of different string states algebraically at each fixed mass level. Thus there is only one independent component of high energy scattering amplitude at each fixed mass level. For the case of string-tree amplitudes, a general formula can even be given to express all high-energy stringy scattering amplitudes for arbitrary mass levels in terms of those of tachyons $[1,3]$. These zero-norm state calculations are independent of the high-energy saddle-point calculations of Gross and Mende [4], Gross [5] and Gross and Manes [6]. In fact, the results of saddle-point calculations by those authors were found [1-3] to be inconsistent with high energy stringy Ward identities of zero-norm state calculations, and thus could threat the validity of unitarity of string perturbation theory. A corrected saddle-point calculation was given in [3], where the missing terms of the calculations in Refs $[4,5,6]$ were identified to recover the stringy Ward identities.

The importance of zero-norm states and their implication on stringy symmetries were first pointed out in the context of massive $\sigma$-model approach of string theory [7]. Some implications of the corresponding stringy Ward identities on the scattering amplitudes were discussed in [8]. On the other hand, zero-norm states were also shown [9] to carry the spacetime $\omega_{\infty}$ symmetry [10] charges of 2D string theory [11]. This is in parallel with the work of [12] where the ground ring structure of ghost number zero operators was identified in the BRST quantization. All the above interesting results of 26D and 2D string theories strongly suggest that a clearer understanding of zero-norm states holds promise to uncover the fundamental symmetry of string theory. Recently, a simplified method to generate zero-norm states in 26D OCFQ bosonic string was proposed [13]. Based on a simplified prescription to calculate positive-norm propagating states given in [14], general formulas of some zero-norm tensor states at arbitrary mass levels were calculated. Unfortunately, general formulas for the complete set of zero-norm states are still lacking mostly due to the high dimensionality of spacetime $\mathrm{D}=26$. However, in the $2 \mathrm{D}$ OCFQ string theory, a general formula of zero- 
norm states with discrete Polyakov's momenta at arbitrary mass levels was given in terms of Schur Polynomials [9]. On the other hand, for the case of 26D string, the background ghost transformations in the gauge transformations of WSFT [15] were shown [16] to correspond, in a one-to-one manner, to the lifting of on-shell conditions of zero-norm states in the OCFQ approach.

In this paper, we shall calculate and identify the counterparts of zero-norm states in two other quantization schemes of 26D open bosonic string theory, namely the light-cone DDF [17] zero-norm states and the off-shell BRST zero-norm states (with ghost) in WSFT. In particular, special attention is paid to the inter-particle zero-norm states in all quantization schemes. For the case of off-shell BRST zero-norm states, we impose the no ghost conditions and recover exactly two types of on-shell zero-norm states in the OCFQ string spectrum for the first few low-lying mass levels. We then show that off-shell gauge transformations of WSFT are identical to the on-shell stringy gauge symmetries generated by two typse of zeronorm states in the OCFQ string theory. Our calculations in this paper serve as the first step to study stringy symmetries in light-cone DDF and BRST string theories, and to bridge the links between different quantization schemes for both on-shell and off-shell string theories. In section II, we first review the calculations of zero-norm states in OCFQ spectrum. The most general spectrum analysis in the helicity basis, including zero-norm states, was then given to discuss the inter-particle $D_{2}$ zero-norm state $[7,8]$ at mass level $m^{2}=4$. We will see that one can use polarization of either one of the two positive-norm states to represent the polarization of the inter-particle zero-norm state. This justifies how one can have the inter-particle symmetry transformation for the two massive modes in the weak field massive $\sigma$-model calculation derived previously [7]. In section III, we calculate both type I and type II zero-norm states in the light-cone DDF string up to mass level $m^{2}=4$. In section IV, we first calculate off-shell zero-norm states with ghosts from linearized gauge transformation of WSFT. After imposing the no ghost conditions on these zero-norm states, we can reproduce exactly two types of zero-norm states in OCFQ spectrum for the first few low-lying mass levels. We then show that off-shell gauge transformations of WSFT are identical to the onshell stringy gauge symmetries generated by two typse of zero-norm states in the generalized massive $\sigma$-model approach [7] of string theory. The high energy limit of these stringy gauge symmetries was recently used to calculate the proportionality constants among high energy scattering amplitudes of different string states conjectured by Gross [5]. Based on the zero- 
norm state calculations [1-3], we have thus related gauge symmetry of WSFT [15] to the high-energy stringy symmetry conjectured by Gross [4-6]. Finally, a brief conclusion is given in section $\mathrm{V}$.

\section{ZERO-NORM STATES IN THE OCFQ SPECTRUM}

In the OCFQ spectrum of open bosonic string theory, the solutions of physical states conditions include positive-norm propagating states and two types of zero-norm states. The latter are $[18]$

Type I : $L_{-1}|x\rangle$, where $L_{1}|x\rangle=L_{2}|x\rangle=0, L_{0}|x\rangle=0$;

Type II : $\left(L_{-2}+\frac{3}{2} L_{-1}^{2}\right)|\widetilde{x}\rangle$, where $L_{1}|\widetilde{x}\rangle=L_{2}|\widetilde{x}\rangle=0,\left(L_{0}+1\right)|\widetilde{x}\rangle=0$.

Eqs.(2.1) and (2.2) can be derived from Kac determinant in conformal field theory. While type I states have zero-norm at any spacetime dimension, type II states have zero-norm only at $\mathrm{D}=26$.

\section{A. Zero-norm states with constraints}

The solutions of Eqs.(2.1) and (2.2) up to the mass level $m^{2}=4$ are listed in the following [13]:

1. $m^{2}=-k^{2}=0$ :

$$
L_{-1}|x\rangle=k \cdot \alpha_{-1}|0, k\rangle ;|x\rangle=|0, k\rangle ;|x\rangle=|0, k\rangle .
$$

2. $m^{2}=-k^{2}=2$ :

$$
\begin{gathered}
\left(L_{-2}+\frac{3}{2} L_{-1}^{2}\right)|\widetilde{x}\rangle=\left[\frac{1}{2} \alpha_{-1} \cdot \alpha_{-1}+\frac{5}{2} k \cdot \alpha_{-2}+\frac{3}{2}\left(k \cdot \alpha_{-1}\right)^{2}\right]|0, k\rangle ;|\widetilde{x}\rangle=|0, k\rangle \\
L_{-1}|x\rangle=\left[\theta \cdot \alpha_{-2}+\left(k \cdot \alpha_{-1}\right)\left(\theta \cdot \alpha_{-1}\right)\right]|0, k\rangle ;|x\rangle=\theta \cdot \alpha_{-1}|0, k\rangle, \theta \cdot k=0 .
\end{gathered}
$$

3. $m^{2}=-k^{2}=4$ : 


$$
\begin{aligned}
& \left(L_{-2}+\frac{3}{2} L_{-1}^{2}\right)|\widetilde{x}\rangle=\left\{4 \theta \cdot \alpha_{-3}+\frac{1}{2}\left(\alpha_{-1} \cdot \alpha_{-1}\right)\left(\theta \cdot \alpha_{-1}\right)+\frac{5}{2}\left(k \cdot \alpha_{-2}\right)\left(\theta \cdot \alpha_{-1}\right)\right. \\
& \left.+\frac{3}{2}\left(k \cdot \alpha_{-1}\right)^{2}\left(\theta \cdot \alpha_{-1}\right)+3\left(k \cdot \alpha_{-1}\right)\left(\theta \cdot \alpha_{-2}\right)\right\}|0, k\rangle ; \\
& |\widetilde{x}\rangle=\theta \cdot \alpha_{-1}|0, k\rangle, k \cdot \theta=0, \\
& L_{-1}|x\rangle=\left[2 \theta_{\mu \nu} \alpha_{-1}^{\mu} \alpha_{-2}^{\nu}+k_{\lambda} \theta_{\mu \nu} \alpha_{-1}^{\lambda} \alpha_{-1}^{\mu} \alpha_{-1}^{\nu}\right]|0, k\rangle ; \\
& |x\rangle=\theta_{\mu \nu} \alpha_{-1}^{\mu \nu}|0, k\rangle, k \cdot \theta=\eta^{\mu \nu} \theta_{\mu \nu}=0, \theta_{\mu \nu}=\theta_{\nu \mu}, \\
& L_{-1}|x\rangle=\left[\frac{1}{2}\left(k \cdot \alpha_{-1}\right)^{2}\left(\theta \cdot \alpha_{-1}\right)+2 \theta \cdot \alpha_{-3}+\frac{3}{2}\left(k \cdot \alpha_{-1}\right)\left(\theta \cdot \alpha_{-2}\right)\right. \\
& \left.+\frac{1}{2}\left(k \cdot \alpha_{-2}\right)\left(\theta \cdot \alpha_{-1}\right)\right]|0, k\rangle \\
& |x\rangle=\left[2 \theta \cdot \alpha_{-2}+\left(k \cdot \alpha_{-1}\right)\left(\theta \cdot \alpha_{-1}\right)\right]|0, k\rangle, \theta \cdot k=0, \\
& L_{-1}|x\rangle=\left[\frac{17}{4}\left(k \cdot \alpha_{-1}\right)^{3}+\frac{9}{2}\left(k \cdot \alpha_{-1}\right)\left(\alpha_{-1} \cdot \alpha_{-1}\right)+9\left(\alpha_{-1} \cdot \alpha_{-2}\right)\right. \\
& \left.+21\left(k \cdot \alpha_{-1}\right)\left(k \cdot \alpha_{-2}\right)+25\left(k \cdot \alpha_{-3}\right)\right]|0, k\rangle ; \\
& |x\rangle=\left[\frac{25}{2} k \cdot \alpha_{-2}+\frac{9}{2} \alpha_{-1} \cdot \alpha_{-1}+\frac{17}{4}\left(k \cdot \alpha_{-1}\right)^{2}\right]|0, k\rangle \text {. }
\end{aligned}
$$

Note that there are two degenerate vector zero-norm states, Eq.(2.6) for type II and Eq.(2.8) for type I, at mass level $m^{2}=4$. We define $D_{2}$ vector zero-norm state by antisymmetrizing those terms which contain $\alpha_{-1}^{\mu} \alpha_{-2}^{\nu}$ in Eqs.(2.6) and (2.8) as following [7]

$$
\left|D_{2}\right\rangle=\left[\left(\frac{1}{2} k_{\mu} k_{\nu} \theta_{\lambda}+2 \eta_{\mu \nu} \theta_{\lambda}\right) \alpha_{-1}^{\mu} \alpha_{-1}^{\nu} \alpha_{-1}^{\lambda}+9 k_{\mu} \theta_{\nu} \alpha_{-2}^{[\mu} \alpha_{-1}^{\nu]}-6 \theta_{\mu} \alpha_{-3}^{\mu}\right]|0, k\rangle, \quad k \cdot \theta=0 .
$$

Similarly $D_{1}$ vector zero-norm state is defined by symmetrizing those terms which contain $\alpha_{-1}^{\mu} \alpha_{-2}^{\nu}$ in Eqs.(2.6) and (2.8)

$$
\left|D_{1}\right\rangle=\left[\left(\frac{5}{2} k_{\mu} k_{\nu} \theta_{\lambda}+\eta_{\mu \nu} \theta_{\lambda}\right) \alpha_{-1}^{\mu} \alpha_{-1}^{\nu} \alpha_{-1}^{\lambda}+9 k_{\mu} \theta_{\nu} \alpha_{-2}^{(\mu} \alpha_{-1}^{\nu)}+6 \theta_{\mu} \alpha_{-3}^{\mu}\right]|0, k\rangle, \quad k \cdot \theta=0 .
$$


In the generalized massive $\sigma$-model approach of string theory, it can be shown that each zero-norm state in the OCFQ spectrum generates a massive symmetry transformation for the propagating string modes. In particular, the inter-particle symmetry transformation corresponding to the $D_{2}$ inter-particle zero-norm state in Eq.(2.10) can be calculated to be $[7]$

$$
\delta C_{(\mu \nu \lambda)}=\left(\frac{1}{2} \partial_{(\mu} \partial_{\nu} \theta_{\lambda)}-2 \eta_{(\mu \nu} \theta_{\lambda)}\right), \delta C_{[\mu \nu]}=9 \partial_{[\mu} \theta_{\nu]},
$$

where $\partial_{\nu} \theta^{\nu}=0,\left(\partial^{2}-4\right) \theta^{\nu}=0$ are the on-shell conditions of the $D_{2}$ vector zero-norm state. $C_{(\mu \nu \lambda)}$ and $C_{[\mu \nu]}$ in Eq.(2.12) are the background fields of the symmetric spin-three and

antisymmetric spin-two states, respectively, at mass level $m^{2}=4$. Eq.(2.12) is the result of the first order weak field approximation but, in contrast to the high energy $\alpha^{\prime} \rightarrow \infty$ result of [1-3], valid to all energy $\alpha^{\prime}$ in the generalized $\sigma$-model approach. It is important to note that the decoupling of $D_{2}$ vector zero-norm state implies simultaneous change of both $C_{(\mu \nu \lambda)}$ and $C_{[\mu \nu]}$, thus they form a gauge multiplet. In general, an inter-particle zero-norm state can be defined to be $D_{2}+\alpha D_{1}$, where $\alpha$ is an arbitrary constant.

\section{B. Zero-norm states in the helicity basis}

In this subsection, we are going to do the most general spectrum analysis which naturally includes zero-norm states. We will then solve the Virasoro constraints in the helicity basis and recover the zero-norm states listed above $[7,13]$. In particular, this analysis will make it clear how $D_{2}$ zero-norm state in Eq.(2.10) can induce the inter-particular symmetry transformation for two propagating states at the mass level $m^{2}=4$.

We begin our discussion for the mass level $m^{2}=2$. At this mass level, the general expression for the physical states can be written as

$$
\left[\epsilon_{\mu \nu} \alpha_{-1}^{\mu} \alpha_{-1}^{\nu}+\epsilon_{\mu} \alpha_{-2}^{\mu}\right]|0, k\rangle
$$

In the OCFQ of string theory, physical states satisfy the mass shell condition

$$
\left(L_{0}-1\right)|p h y s\rangle=0 \Rightarrow k^{2}=-2
$$


and the Virasoro constraints $L_{1}|p h y s\rangle=L_{2}|p h y s\rangle=0$ which give

$$
\begin{aligned}
\epsilon_{\mu} & =-\epsilon_{\mu \nu} k^{\nu}, \\
\eta^{\mu \nu} \epsilon_{\mu \nu} & =2 \epsilon_{\mu \nu} k^{\mu} k^{\nu} .
\end{aligned}
$$

In order to solve for the constraints Eq.(2.15) and Eq.(2.16) in a covariant way, it is convenient to make the following change of basis,

$$
\begin{aligned}
e_{P} & \equiv \frac{1}{m}(E, 0, \ldots, \mathrm{k}) \\
e_{L} & \equiv \frac{1}{m}(\mathrm{k}, 0, \ldots ., E) \\
e_{T_{i}} & \equiv(0,0, \ldots ., 1(\mathrm{i} \text {-th spatial direction }), \ldots, 0), \quad i=1,2, \ldots, 24 .
\end{aligned}
$$

The 2nd rank tensor $\epsilon_{\mu \nu}$ can be written in the helicity basis Eqs.(2.17)-(2.19) as

$$
\epsilon_{\mu \nu}=\sum_{A, B} u_{A B} e_{\mu}^{A} e_{\nu}^{B}, \quad A, B=P, L, T_{i} .
$$

In this new representation, the second Virasoro constraint Eq.(2.16) reduces to a simple algebraic relation, and one can solve it

$$
u_{P P}=\frac{1}{5}\left(u_{L L}+\sum_{i=1}^{24} u_{T_{i} T_{i}}\right) .
$$

In order to perform an irreducible decomposition of the spin-two state into the trace and traceless parts, we define the following variables

$$
\begin{aligned}
& x \equiv \frac{1}{25}\left(u_{L L}+\sum_{i=1}^{24} u_{T_{i} T_{i}}\right), \\
& y \equiv \frac{1}{25}\left(u_{L L}-\frac{1}{24} \sum_{i=1}^{24} u_{T_{i} T_{i}}\right) .
\end{aligned}
$$


We can then write down the complete decompositions of the spin-two polarization tensor as

$$
\begin{aligned}
\epsilon_{\mu \nu} & =x\left(5 e_{\mu}^{P} e_{\nu}^{P}+e_{\mu}^{L} e_{\nu}^{L}+\sum_{i=1}^{24} e_{\mu}^{T_{i}} e_{\nu}^{T_{i}}\right) \\
& +y \sum_{i=1}^{24}\left(e_{\mu}^{L} e_{\nu}^{L}-e_{\mu}^{T_{i}} e_{\nu}^{T_{i}}\right) \\
& +\sum_{i, j}\left(u_{T_{i} T_{j}}-\frac{\delta_{i j}}{24} \sum_{l=1}^{24} u_{T_{l} T_{l}}\right) e_{\mu}^{T_{i}} e_{\nu}^{T_{j}} \\
& +u_{P L}\left(e_{\mu}^{P} e_{\nu}^{L}+e_{\mu}^{L} e_{\nu}^{P}\right) \\
& +\sum_{i=1}^{24} u_{P T_{i}}\left(e_{\mu}^{P} e_{\nu}^{T_{i}}+e_{\mu}^{T_{i}} e_{\nu}^{P}\right) \\
& +\sum_{i=1}^{24} u_{L T_{i}}\left(e_{\mu}^{L} e_{\nu}^{T_{i}}+e_{\mu}^{T_{i}} e_{\nu}^{L}\right) .
\end{aligned}
$$

The first Virasoro constraint Eq.(2.15) implies that $\epsilon_{\mu}$ vector is not an independent variable, and is related to the spin-two polarization tensor $\epsilon_{\mu \nu}$ as follows

$$
\epsilon_{\mu}=5 \sqrt{2} x e_{\mu}^{P}+\sqrt{2} u_{P L} e_{\mu}^{L}+\sqrt{2} \sum_{i=1}^{24} u_{P T_{i}} e_{\mu}^{T_{i}} .
$$

Finally, combining the results of Eqs.(2.13),(2.24) and (2.25), we get the complete solution for physical states at mass level $m^{2}=2$

$$
\begin{aligned}
& {\left[\epsilon_{\mu \nu} \alpha_{-1}^{\mu} \alpha_{-1}^{\nu}+\epsilon_{\mu} \alpha_{-2}^{\mu}\right]|0, k\rangle} \\
& =x\left(5 \alpha_{-1}^{P} \alpha_{-1}^{P}+\alpha_{-1}^{L} \alpha_{-1}^{L}+\sum_{i=1}^{24} \alpha_{-1}^{T_{i}} \alpha_{-1}^{T_{i}}+5 \sqrt{2} \alpha_{-2}^{P}\right)|0, k\rangle \\
& +y \sum_{i=1}^{24}\left(\alpha_{-1}^{L} \alpha_{-1}^{L}-\alpha_{-1}^{T_{i}} \alpha_{-1}^{T_{i}}\right)|0, k\rangle \\
& +\sum_{i, j}\left(u_{T_{i} T_{j}}-\frac{\delta_{i j}}{24} \sum_{l=1}^{24} u_{T_{l} T_{l}}\right) \alpha_{-1}^{T_{i}} \alpha_{-1}^{T_{j}}|0, k\rangle \\
& +u_{P L}\left(2 \alpha_{-1}^{P} \alpha_{-1}^{L}+\sqrt{2} \alpha_{-2}^{L}\right)|0, k\rangle \\
& +\sum_{i=1}^{24} u_{P T_{i}}\left(2 \alpha_{-1}^{P} \alpha_{-1}^{T_{i}}+\sqrt{2} \alpha_{-2}^{T_{i}}\right)|0, k\rangle \\
& +2 \sum_{i=1}^{24} u_{L T_{i}} \alpha_{-1}^{L} \alpha_{-1}^{T_{i}}|0, k\rangle,
\end{aligned}
$$


where the oscillator creation operators $\alpha_{-1}^{P}, \alpha_{-1}^{L}, \alpha_{-1}^{T_{i}}$, etc., are defined as

$$
\alpha_{-n}^{A} \equiv e_{\mu}^{A} \cdot \alpha_{-n}^{\mu}, \quad n \in N, \quad A=P, L, T_{i} .
$$

In comparison with the standard expressions for zero-norm states in subsection A, we find that Eqs. (2.26), (2.29) and (2.30) are identical to the type II singlet and type I vector zero-norm states for the mass level $m^{2}=2$

$$
\begin{aligned}
& (2.26)=2 x\left[\left(\frac{1}{2} \eta_{\mu \nu}+\frac{3}{2} k_{\mu} k_{\nu}\right) \alpha_{-1}^{\mu} \alpha_{-1}^{\nu}+\frac{5}{2} k_{\mu} \alpha_{-2}^{\mu}\right]|0, k\rangle, \\
& (2.29)=\sqrt{2} u_{P L}\left[e_{\mu}^{L} k_{\nu} \alpha_{-1}^{\mu} \alpha_{-1}^{\nu}+e_{\mu}^{L} \alpha_{-2}^{\mu}\right]|0, k\rangle, \\
& (2.30)=\sum_{i=1}^{24} \sqrt{2} u_{P T_{i}}\left[e_{\mu}^{T_{i}} k_{\nu} \alpha_{-1}^{\mu} \alpha_{-1}^{\nu}+e_{\mu}^{T_{i}} \alpha_{-2}^{\mu}\right]|0, k\rangle .
\end{aligned}
$$

In addition, one can clearly see from our covariant decomposition how zero-norm states generate gauge transformations on positve-norm states. While a nonzero value for $x$ induces a gauge transformation along the type II singlet zero-norm state direction, the coefficients $u_{P L}, u_{P T_{i}}$ parametrize the type I vector gauge transformations with polarization vectors $\theta=e^{L}$ and $\theta=e^{T_{i}}$, respectively. Finally, by a simple counting of degrees of freedom, one can identify Eqs (2.27), (2.28) and (2.31) as the singlet (1), (traceless) tensor (299), and vector (24) positive-norm states, respectively. These positive-norm states are in a one-to-one correspondence with the degrees of freedom in the light-cone quantization scheme.

We now turn to the analysis of $m^{2}=4$ spectrum. Due to the complexity of our calculations, we shall present the calculations in three steps. We shall first write down all of physical states (including both positive-norm and zero-norm states) in the simplest gauge choices in the helicity basis. We then calculate the spin-3 state decomposition in the most general gauge choice. Finally, the complete analysis will be given to see how $D_{2}$ zero-norm state in Eq.(2.10) can induce the inter-particle symmetry transformation for two propagating states at the mass level $m^{2}=4$.

\section{Physical states in the simplest gauge choices}

To begin with, let us first analyse the positive-norm states. There are two particles at the mass level $m^{2}=4$, a totally symmetric spin-three particle and an antisymmetric spin-two particle. The canonical representation of the spin-three state is usually choosen as

$$
\epsilon_{\mu \nu \lambda} \alpha_{-1}^{\mu} \alpha_{-1}^{\nu} \alpha_{-1}^{\lambda}|0, k\rangle, \quad k^{2}=-4,
$$


where the totally symmetric polarization tensor $\epsilon_{\mu \nu \lambda}$ can be expanded in the helicity basis as

$$
\epsilon_{\mu \nu \lambda}=\sum_{A, B, C} \tilde{u}_{A B C} e_{\mu}^{A} e_{\nu}^{B} e_{\lambda}^{C}, \quad A, B, C=P, L, T_{i}
$$

The Virasoro conditions on the polarization tensor can be solved as follows

$$
\begin{aligned}
& k^{\lambda} \epsilon_{\mu \nu \lambda}=0 \Rightarrow \tilde{u}_{P A B}=0, \quad \forall A, B=P, L, T_{i}, \\
& \eta^{\nu \lambda} \epsilon_{\mu \nu \lambda}=0 \Rightarrow \tilde{u}_{L L L}+\sum_{i} \tilde{u}_{T_{i} T_{i} L}=0, \\
& \tilde{u}_{L L T_{i}}+\sum_{j} \tilde{u}_{T_{j} T_{j} T_{i}}=0 .
\end{aligned}
$$

If we choose to keep the minimal number of $L$ components in the expansion coefficients $\tilde{u}_{A B C}$ for the spin-three particle, we get the following canonical decomposition

$$
\begin{aligned}
|A(\epsilon)\rangle \equiv & \left(\epsilon_{\mu \nu \lambda} \alpha_{-1}^{\mu} \alpha_{-1}^{\nu} \alpha_{-1}^{\lambda}\right)|0, k\rangle=|A(\tilde{u})\rangle \\
& =\sum_{i} \tilde{u}_{T_{i} T_{i} T_{i}}\left(\alpha_{-1}^{T_{i}} \alpha_{-1}^{T_{i}} \alpha_{-1}^{T_{i}}-3 \alpha_{-1}^{L} \alpha_{-1}^{L} \alpha_{-1}^{T_{i}}\right)|0, k\rangle \\
& +\sum_{i \neq j} 3 \tilde{u}_{T_{j} T_{j} T_{i}}\left(\alpha_{-1}^{T_{j}} \alpha_{-1}^{T_{j}} \alpha_{-1}^{T_{i}}-\alpha_{-1}^{L} \alpha_{-1}^{L} \alpha_{-1}^{T_{i}}\right)|0, k\rangle \\
& +\sum_{(i \neq j \neq k)} 6 \tilde{u}_{T_{i} T_{j} T_{k}}\left(\alpha_{-1}^{T_{i}} \alpha_{-1}^{T_{j}} \alpha_{-1}^{T_{k}}\right)|0, k\rangle \\
& +\sum_{i} \tilde{u}_{L T_{i} T_{i}}\left(3 \alpha_{-1}^{L} \alpha_{-1}^{T_{i}} \alpha_{-1}^{T_{i}}-\alpha_{-1}^{L} \alpha_{-1}^{L} \alpha_{-1}^{L}\right)|0, k\rangle \\
& +\sum_{(i \neq j)} 6 \tilde{u}_{L T_{i} T_{j}}\left(\alpha_{-1}^{L} \alpha_{-1}^{T_{i}} \alpha_{-1}^{T_{j}}\right)|0, k\rangle .
\end{aligned}
$$

It is easy to check that the 2900 independent degrees of freedom of the spin-three particle decompose into $24+552+2024+24+276$ in the above representation.

Similarily, for the antisymmetric spin-two particle, we have the following canonical representation

$$
\epsilon_{[\mu, \nu]} \alpha_{-1}^{\mu} \alpha_{-2}^{\nu}|0, k\rangle
$$

Rewriting the polarization tensor $\epsilon_{[\mu, \nu]}$ in the helicity basis

$$
\epsilon_{[\mu, \nu]}=\sum_{A, B} v_{[A, B]} e_{\mu}^{A} e_{\nu}^{B}
$$


and solving the Virasoro constraints

$$
k^{\nu} \epsilon_{[\mu, \nu]}=2 v_{[P, L]} e_{\mu}^{L}+2 \sum_{i=1}^{24} v_{\left[P, T_{i}\right]} e_{\mu}^{T_{i}}=0,
$$

we obtain the following decomposition for the spin-two state

$$
\begin{aligned}
|B(\epsilon)\rangle \equiv & \epsilon_{[\mu, \nu]} \alpha_{-1}^{\mu} \alpha_{-2}^{\nu}|0, k\rangle=|B(v)\rangle \\
& =\sum_{i} v_{\left[L, T_{i}\right]}\left(\alpha_{-1}^{L} \alpha_{-2}^{T_{i}}-\alpha_{-1}^{T_{i}} \alpha_{-2}^{L}\right)|0, k\rangle \\
& +\sum_{(i \neq j)} v_{\left[T_{i}, T_{j}\right]}\left(\alpha_{-1}^{T_{i}} \alpha_{-2}^{T_{j}}-\alpha_{-1}^{T_{j}} \alpha_{-2}^{T_{i}}\right)|0, k\rangle .
\end{aligned}
$$

Finally, one can check that the 300 independent degrees of freedom of the spin-two particle decompose into $24+276$ in the above expression.

For the zero-norm states at $m^{2}=4$, we have the following decompositions

1. Spin-two tensor

$$
\begin{aligned}
|C(\theta)\rangle & \equiv\left(k_{\lambda} \theta_{\mu \nu} \alpha_{-1}^{\mu} \alpha_{-1}^{\nu} \alpha_{-1}^{\lambda}+2 \theta_{\mu \nu} \alpha_{-1}^{\mu} \alpha_{-2}^{\nu}\right)|0, k\rangle \\
& =\sum_{i} 2 \theta_{T_{i} T_{i}}\left(\alpha_{-1}^{T_{i}} \alpha_{-1}^{T_{i}} \alpha_{-1}^{P}-\alpha_{-1}^{L} \alpha_{-1}^{L} \alpha_{-1}^{P}+\alpha_{-1}^{T_{i}} \alpha_{-2}^{T_{i}}-\alpha_{-1}^{L} \alpha_{-2}^{L}\right)|0, k\rangle \\
& +\sum_{(i \neq j)} 2 \theta_{T_{i} T_{j}}\left(2 \alpha_{-1}^{T_{i}} \alpha_{-1}^{T_{j}} \alpha_{-1}^{P}+\alpha_{-1}^{T_{i}} \alpha_{-2}^{T_{j}}+\alpha_{-1}^{T_{j}} \alpha_{-2}^{T_{i}}\right)|0, k\rangle \\
& +\sum_{i} 2 \theta_{L T_{i}}\left(2 \alpha_{-1}^{L} \alpha_{-1}^{T_{i}} \alpha_{-1}^{P}+\alpha_{-1}^{L} \alpha_{-2}^{T_{i}}+\alpha_{-1}^{T_{i}} \alpha_{-2}^{L}\right)|0, k\rangle,
\end{aligned}
$$

where we have solved the Virasoro constraints on the polarization tensor $\theta_{\mu \nu}$

$$
\begin{aligned}
\theta_{\mu \nu} & =\sum_{A, B} \theta_{A B} e_{\mu}^{A} e_{\nu}^{B}, \\
\eta^{\mu \nu} \theta_{\mu \nu} & =-\theta_{P P}+\theta_{L L}+\sum_{i} \theta_{T_{i} T_{i}}=0 \\
k^{\nu} \theta_{\mu \nu} & =-2 \theta_{P P} e_{\mu}^{P}-2 \theta_{P L} e_{\nu}^{L}-2 \sum_{i} \theta_{P T_{i}} e_{\mu}^{T_{i}}=0 .
\end{aligned}
$$

The 324 degrees of freedom of on-shell $\theta_{\mu \nu}$ decompose into $24+276+24$ in Eq.(2.43). 
2. Spin-one vector (with polarization vector $\theta \cdot k=0, \theta_{\mu}=\sum_{A} \theta_{A} e_{\mu}^{A}, A=L, T_{i}$ )

$$
\begin{aligned}
\left|D_{1}(\theta)\right\rangle & \equiv\left[\left(\frac{5}{2} k_{\mu} k_{\nu} \theta_{\lambda}+\eta_{\mu \nu} \theta_{\lambda}\right) \alpha_{-1}^{\mu} \alpha_{-1}^{\nu} \alpha_{-1}^{\lambda}\right. \\
& \left.+9 k_{(\mu} \theta_{\nu)} \alpha_{-1}^{\mu} \alpha_{-2}^{\nu}+6 \theta_{\mu} \alpha_{-3}^{\mu}\right]|0, k\rangle \\
& =\sum_{A} \theta_{A}\left[9 \alpha_{-1}^{P} \alpha_{-1}^{P} \alpha_{-1}^{A}+\alpha_{-1}^{L} \alpha_{-1}^{L} \alpha_{-1}^{A}+\sum_{i} \alpha_{-1}^{T_{i}} \alpha_{-1}^{T_{i}} \alpha_{-1}^{A}\right. \\
& \left.+9\left(\alpha_{-1}^{P} \alpha_{-2}^{A}+\alpha_{-1}^{A} \alpha_{-2}^{P}\right)+6 \alpha_{-3}^{A}\right]|0, k\rangle .
\end{aligned}
$$

3. Spin-one vector (with polarization vector $\theta \cdot k=0, \theta_{\mu}=\sum_{A} \theta_{A} e_{\mu}^{A}, A=L, T_{i}$ )

$$
\begin{aligned}
\left|D_{2}(\theta)\right\rangle & \equiv\left[\left(\frac{1}{2} k_{\mu} k_{\nu} \theta_{\lambda}+2 \eta_{\mu \nu} \theta_{\lambda}\right) \alpha_{-1}^{\mu} \alpha_{-1}^{\nu} \alpha_{-1}^{\lambda}\right. \\
& \left.-9 k_{[\mu} \theta_{\nu]} \alpha_{-1}^{\mu} \alpha_{-2}^{\nu}-6 \theta_{\mu} \alpha_{-3}^{\mu}\right]|0, k\rangle \\
& =\sum_{A} \theta_{A}\left[2 \alpha_{-1}^{L} \alpha_{-1}^{L} \alpha_{-1}^{A}+2 \sum_{j} \alpha_{-1}^{T_{j}} \alpha_{-1}^{T_{j}} \alpha_{-1}^{A}\right. \\
& \left.-9\left(\alpha_{-1}^{P} \alpha_{-2}^{A}-\alpha_{-1}^{A} \alpha_{-2}^{P}\right)-6 \alpha_{-3}^{A}\right]|0, k\rangle .
\end{aligned}
$$

4. spin-zero singlet

$$
\begin{aligned}
|E\rangle & \equiv\left[\left(\frac{17}{4} k_{\mu} k_{\nu} k_{\lambda}+\frac{9}{2} \eta_{\mu \nu} k_{\lambda}\right) \alpha_{-1}^{\mu} \alpha_{-1}^{\nu} \alpha_{-1}^{\lambda}\right. \\
& \left.+\left(21 k_{\mu} k_{\nu}+9 \eta_{\mu \nu}\right) \alpha_{-1}^{\mu} \alpha_{-2}^{\nu}+25 k_{\mu} \alpha_{-3}^{\mu}\right]|0, k\rangle \\
& =\left[25\left(\alpha_{-1}^{P} \alpha_{-1}^{P} \alpha_{-1}^{P}+3 \alpha_{-1}^{P} \alpha_{-2}^{P}+2 \alpha_{-3}^{P}\right)\right. \\
& \left.+9 \alpha_{-1}^{L} \alpha_{-1}^{L} \alpha_{-1}^{P}+9 \alpha_{-1}^{L} \alpha_{-2}^{L}+9 \sum_{i}\left(\alpha_{-1}^{T_{i}} \alpha_{-1}^{T_{i}} \alpha_{-1}^{P}+\alpha_{-1}^{T_{i}} \alpha_{-2}^{T_{i}}\right)\right]|0, k\rangle .
\end{aligned}
$$

2. Spin-three state in the most general gauge choice

In this subsection, we study the most general gauge choice associated with the totally symmetric spin-three state

$$
\left[\varepsilon_{\mu \nu \lambda} \alpha_{-1}^{\mu} \alpha_{-1}^{\nu} \alpha_{-1}^{\lambda}+\varepsilon_{(\mu \nu)} \alpha_{-1}^{\mu} \alpha_{-2}^{\nu}+\varepsilon_{\mu} \alpha_{-3}^{\mu}\right]|0, k\rangle
$$


where Virasoro constraints imply

$$
\begin{aligned}
\varepsilon_{(\mu \nu)} & =-\frac{3}{2} k^{\lambda} \varepsilon_{\mu \nu \lambda,}, \\
\varepsilon_{\mu} & =\frac{1}{2} k^{\nu} k^{\lambda} \varepsilon_{\mu \nu \lambda}, \\
2 \eta^{\mu \nu} \varepsilon_{\mu \nu \lambda} & =k^{\mu} k^{\nu} \varepsilon_{\mu \nu \lambda} .
\end{aligned}
$$

Eqs.(2.54) and (2.55) imply that both $\varepsilon_{(\mu \nu)}$ and $\varepsilon_{\mu}$ are not independent variables, and Eq.(2.56) stands for the constraint on the polarization $\varepsilon_{\mu \nu \lambda}$. In the helicity basis, we define

$$
\varepsilon_{\mu \nu \lambda}=\sum_{A, B, C} u_{A B C} e_{\mu}^{A} e_{\nu}^{B} e_{\lambda}^{C}, \quad A, B, C=P, L, T_{i} .
$$

Eq.(2.56) then gives

$$
\sum_{A, B} \eta^{A B} u_{A B C}=2 u_{P P C}, \quad A, B, C=P, L, T_{i},
$$

which implies

$$
3 u_{P P C}-u_{L L C}-\sum_{j} u_{T_{j} T_{j} C}=0, \quad C=P, L, T_{i} .
$$


Eliminating $u_{L L P}, u_{L L L}$ and $u_{L L T_{i}}$ from above equations, we have the solution for $\varepsilon_{\mu \nu \lambda}, \varepsilon_{(\mu \nu)}$ and $\varepsilon_{\mu}$

$$
\begin{aligned}
& \varepsilon_{\mu \nu \lambda}=u_{P P P}\left[e_{\mu}^{P} e_{\nu}^{P} e_{\lambda}^{P}+3\left(e_{\mu}^{L} e_{\nu}^{L} e_{\lambda}^{P}+\text { per. }\right)\right] \\
& +u_{P P L}\left[\left(e_{\mu}^{P} e_{\nu}^{P} e_{\lambda}^{L}+\text { per. }\right)+3 e_{\mu}^{L} e_{\nu}^{L} e_{\lambda}^{L}\right] \\
& +\sum_{i} u_{P P T_{i}}\left[\left(e_{\mu}^{P} e_{\nu}^{P} e_{\lambda}^{T_{i}}+\text { per. }\right)+3\left(e_{\mu}^{L} e_{\nu}^{L} e_{\lambda}^{T_{i}}+\text { per. }\right)\right] \\
& +\sum_{i} u_{P T_{i} T_{i}}\left[\left(e_{\mu}^{P} e_{\nu}^{T_{i}} e_{\lambda}^{T_{i}}+\text { per. }\right)-\left(e_{\mu}^{L} e_{\nu}^{L} e_{\lambda}^{P}+\text { per. }\right)\right] \\
& +\sum_{(i \neq j)} u_{P T_{i} T_{j}}\left[e_{\mu}^{P} e_{\nu}^{T_{i}} e_{\lambda}^{T_{j}}+\text { per. }\right] \\
& +\sum_{i} u_{P L T_{i}}\left[e_{\mu}^{P} e_{\nu}^{L} e_{\lambda}^{T_{i}}+\text { per. }\right] \\
& +\sum_{i} u_{L T_{i} T_{i}}\left[\left(e_{\mu}^{L} e_{\nu}^{T_{i}} e_{\lambda}^{T_{i}}+\text { per. }\right)-e_{\mu}^{L} e_{\nu}^{L} e_{\lambda}^{L}\right] \\
& +\sum_{(i \neq j)} u_{L T_{i} T_{j}}\left[e_{\mu}^{L} e_{\nu}^{T_{i}} e_{\lambda}^{T_{j}}+\text { per. }\right] \\
& +\sum_{i} u_{T_{i} T_{i} T_{i}}\left[e_{\mu}^{T_{i}} e_{\nu}^{T_{i}} e_{\lambda}^{T_{i}}-\left(e_{\mu}^{L} e_{\nu}^{L} e_{\lambda}^{T_{i}}+\text { per. }\right)\right] \\
& +\sum_{i \neq j} u_{T_{j} T_{j} T_{i}}\left[\left(e_{\mu}^{T_{j}} e_{\nu}^{T_{j}} e_{\lambda}^{T_{i}}+\text { per. }\right)-\left(e_{\mu}^{L} e_{\nu}^{L} e_{\lambda}^{T_{i}}+\text { per. }\right)\right] \\
& +\sum_{(i \neq j \neq k)} u_{T_{i} T_{j} T_{k}}\left[e_{\mu}^{T_{i}} e_{\nu}^{T_{j}} e_{\lambda}^{T_{k}}+\text { per. }\right] \\
& \frac{1}{3} \varepsilon_{(\mu \nu)}=u_{P P P}\left(e_{\mu}^{P} e_{\nu}^{P}+3 e_{\mu}^{L} e_{\nu}^{L}\right) \\
& +u_{P P L}\left(e_{\mu}^{P} e_{\nu}^{L}+e_{\mu}^{L} e_{\nu}^{P}\right) \\
& +\sum_{i} u_{P P T_{i}}\left(e_{\mu}^{P} e_{\nu}^{T_{i}}+e_{\mu}^{T_{i}} e_{\nu}^{P}\right) \\
& +\sum_{i} u_{P L T_{i}}\left(e_{\mu}^{L} e_{\nu}^{T_{i}}+e_{\mu}^{T_{i}} e_{\nu}^{L}\right) \\
& +\sum_{i} u_{P T_{i} T_{i}}\left(e_{\mu}^{T_{i}} e_{\nu}^{T_{i}}-e_{\mu}^{L} e_{\nu}^{L}\right) \\
& +\sum_{(i \neq j)} u_{P T_{i} T_{j}}\left(e_{\mu}^{T_{i}} e_{\nu}^{T_{j}}+e_{\nu}^{T_{j}} e_{\mu}^{T_{j}}\right) \\
& \frac{1}{2} \varepsilon_{\mu}=\left[u_{P P P} e_{\mu}^{P}+u_{P P L} e_{\mu}^{L}+\sum_{i} u_{P P T_{i}} e_{\mu}^{T_{i}}\right] .
\end{aligned}
$$


Putting all these polarizations back to the general form of physical states Eq.(2.53), we get

$$
\begin{aligned}
& {\left[\varepsilon_{\mu \nu \lambda} \alpha_{-1}^{\mu} \alpha_{-1}^{\nu} \alpha_{-1}^{\lambda}+\varepsilon_{(\mu \nu)} \alpha_{-1}^{\mu} \alpha_{-2}^{\nu}+\varepsilon_{\mu} \alpha_{-3}^{\mu}\right]|0, k\rangle} \\
& =|A(\tilde{u})\rangle+|C(\theta)\rangle \\
& +\left[\frac{1}{9}\left(u_{L L L}+\sum_{i} u_{T_{i} T_{i} L}\right)\right]\left|D_{1}\left(e^{L}\right)\right\rangle \\
& +\sum_{i}\left[\frac{1}{9}\left(u_{L L T_{i}}+\sum_{j} u_{T_{j} T_{j} T_{i}}\right)\right]\left|D_{1}\left(e^{T_{i}}\right)\right\rangle \\
& +\frac{1}{75}\left[u_{L L P}+\sum_{i} u_{P T_{i} T_{i}}\right]|E\rangle .
\end{aligned}
$$

For the first two terms on the right hand side of Eq.(2.63), we need to make the following replacements. For the positive-norm state $|A(\tilde{u})\rangle$ in Eq.(2.38)

$$
\begin{aligned}
& \tilde{u}_{T_{i} T_{i} T_{i}} \rightarrow u_{T_{i} T_{i} T_{i}}-\frac{1}{3} u_{P P T_{i}}, \quad \tilde{u}_{T_{j} T_{j} T_{i}} \rightarrow u_{T_{j} T_{j} T_{i}}-\frac{1}{9} u_{P P T_{i}}, \\
& \tilde{u}_{T_{i} T_{j} T_{k}} \rightarrow u_{T_{i} T_{j} T_{k}} \quad \tilde{u}_{L T_{i} T_{i}} \rightarrow u_{L T_{i} T_{i}}-\frac{1}{9} u_{P P L}, \quad \tilde{u}_{L T_{i} T_{j}} \rightarrow u_{L T_{i} T_{j}} .
\end{aligned}
$$

For the spin-two zero-norm state $|C(\theta)\rangle$ in Eq.(2.43), the replacement is given by

$$
2 \theta_{L T_{i}} \rightarrow 3 u_{P L T_{i}}, \quad 2 \theta_{T_{i} T_{j}} \rightarrow 3 u_{P T_{i} T_{j}} \text {, for } i \neq j, \quad 2 \theta_{T_{i} T_{i}} \rightarrow 3\left(u_{P T_{i} T_{i}}-\frac{3}{25} u_{P P P}\right) .
$$

It is important to note that for the spin-three gauge multiplet, only spin-two, singlet and $D_{1}$ vector zero-norm states appear in the decomposition Eq.(2.63). In the next subsection, we will see how one can include the missing $D_{2}$ zero-norm state in the analysis.

\section{Complete spectrum analysis and the $D_{2}$ zero-norm state}

After all these preparations, we are ready for a complete analysis of the most general decomposition of physical states at $m^{2}=4$. The most general form of physical states at this mass level are given by

$$
\left[\epsilon_{\mu \nu \lambda} \alpha_{-1}^{\mu} \alpha_{-1}^{\nu} \alpha_{-1}^{\lambda}+\epsilon_{(\mu \nu)} \alpha_{-1}^{\mu} \alpha_{-2}^{\nu}+\epsilon_{[\mu \nu]} \alpha_{-1}^{\mu} \alpha_{-2}^{\nu}+\epsilon_{\mu} \alpha_{-3}^{\mu}\right]|0, k\rangle
$$

The Virasoro constraints are

$$
\begin{aligned}
\epsilon_{(\mu \nu)} & =-\frac{3}{2} k^{\lambda} \epsilon_{\mu \nu \lambda}, \\
-k^{\nu} \epsilon_{[\mu \nu]}+3 \epsilon_{\mu} & =\frac{3}{2} k^{\nu} k^{\lambda} \epsilon_{\mu \nu \lambda}, \\
2 k^{\nu} \epsilon_{[\mu \nu]}+3 \epsilon_{\mu} & =3\left(k^{\nu} k^{\lambda}-\eta^{\nu \lambda}\right) \epsilon_{\mu \nu \lambda} .
\end{aligned}
$$


The solutions to Eqs.(2.68) and (2.69) are given by

$$
\begin{aligned}
k^{\nu} \epsilon_{[\mu \nu]} & =\left(\frac{1}{2} k^{\nu} k^{\lambda}-\eta^{\nu \lambda}\right) \epsilon_{\mu \nu \lambda}, \\
3 \epsilon_{\mu} & =\left(2 k^{\nu} k^{\lambda}-\eta^{\nu \lambda}\right) \epsilon_{\mu \nu \lambda} .
\end{aligned}
$$

In contrast to the previous discussion Eqs.(2.54),(2.55), where both $\epsilon_{(\mu \nu)}$ and $\epsilon_{\mu}$ are completely fixed by the leading spin-three polarization tensor $\epsilon_{\mu \nu \lambda}$, we now have a new contribution from $k^{\nu} \epsilon_{[\mu \nu]}$. It will become clear that this extra term includes the interparticle zero-norm state $D_{2}$, Eqs.(2.49) or (2.50). Furthermore, it should be clear that the antisymmetric spin-two positive-norm physical states are defined by requiring $\epsilon_{\mu \nu \lambda}=\epsilon_{(\mu \nu)}=$ 0 and $\epsilon_{\mu}=k^{\nu} \epsilon_{[\mu \nu]}=0$. In the following, for the sake of clarity, we shall focus on the effects of the new contribution induced by the $\epsilon_{[\mu \nu]}$ only.

The two independent polarization tensors of the most general representation for physical states Eq.(2.66) are given in the helicity basis by

$$
\begin{aligned}
\epsilon_{\mu \nu \lambda} & =\sum_{A B C} U_{A B C} e_{\mu}^{A} e_{\nu}^{B} e_{\lambda}^{C}, \quad A, B, C=P, L, T_{i} ; \\
\epsilon_{[\mu \nu]} & =\sum_{A, B} V_{[A B]} e_{\mu}^{A} e_{\nu}^{B} .
\end{aligned}
$$

The Virasoro constraint Eq.(2.70) demands that

$$
\begin{aligned}
3 U_{P P P}-U_{L L P}-\sum_{i} U_{P T_{i} T_{i}} & =0 \\
3 U_{P P L}-U_{L L L}-\sum_{i} U_{L T_{i} T_{i}} & =2 V_{[P L]}, \\
3 U_{P P T_{i}}-U_{L L T_{i}}-\sum_{j} U_{T_{j} T_{j} T_{i}} & =2 V_{\left[P T_{i}\right]} .
\end{aligned}
$$

In contrast to Eq.(2.59), the solution to the above equations become

$$
\begin{gathered}
U_{P P L}=U_{P P L}^{(1)}+U_{P P L}^{(2)}, \text { where } U_{P P L}^{(1)}=\frac{1}{3}\left(U_{L L L}+\sum_{i} U_{T_{i} T_{i} L}\right), U_{P P L}^{(2)}=\frac{2}{3} V_{[P L]} \\
U_{P P T_{i}}=U_{P P T_{i}}^{(1)}+U_{P P T_{i}}^{(2)}, \text { where } U_{P P T_{i}}^{(1)}=\frac{1}{3}\left(U_{L L T_{i}}+\sum_{j} U_{T_{j} T_{j} T_{i}}\right), U_{P P T_{i}}^{(2)}=\frac{2}{3} V_{\left[P T_{i}\right]} .
\end{gathered}
$$

It is clear from the expressions above that only $U_{P P L}^{(2)}$ and $U_{P P T_{i}}^{(2)}$ give new contributions to 
our previous analysis in the last subsection, so we can simply write down all these new terms

$$
\begin{aligned}
\delta \epsilon_{\mu \nu \lambda} & =\frac{2}{3}\left[V_{[P L]}\left(e_{\mu}^{P} e_{\nu}^{P} e_{\lambda}^{L}+\text { per. }\right)+\sum_{i} V_{\left[P T_{i}\right]}\left(e_{\mu}^{P} e_{\nu}^{P} e_{\lambda}^{T_{i}}+\text { per. }\right)\right] \\
\delta \epsilon_{[\mu \nu]} & =V_{[P L]}\left(e_{\mu}^{P} e_{\nu}^{L}-\text { per. }\right)+\sum_{i} V_{\left[P T_{i}\right]}\left(e_{\mu}^{P} e_{\nu}^{T_{i}}-\text { per. }\right) \\
& +\sum_{i} V_{\left[T_{i} L\right]}\left(e_{\mu}^{T_{i}} e_{\nu}^{L}-\text { per. }\right)+\sum_{i \neq j} V_{\left[T_{j} T_{i}\right]}\left(e_{\mu}^{T_{j}} e_{\nu}^{T_{i}}-\text { per. }\right) \\
\delta \epsilon_{(\mu \nu)} & =2\left[V_{[P L]}\left(e_{\mu}^{P} e_{\nu}^{L}+\text { per. }\right)+\sum_{i} V_{\left[P T_{i}\right]}\left(e_{\mu}^{P} e_{\nu}^{T_{i}}+\text { per. }\right)\right] \\
\delta \epsilon_{\mu} & =2\left[V_{[P L]} e_{\mu}^{L}+\sum_{i} V_{\left[P T_{i}\right]} e_{\mu}^{\left.T_{i}\right]}\right.
\end{aligned}
$$

Finally, the complete decomposition of physical states Eq.(2.66) in the helicity basis becomes

$$
\begin{aligned}
& {\left[\epsilon_{\mu \nu \lambda} \alpha_{-1}^{\mu} \alpha_{-1}^{\nu} \alpha_{-1}^{\lambda}+\epsilon_{(\mu \nu)} \alpha_{-1}^{\mu} \alpha_{-2}^{\nu}+\epsilon_{[\mu \nu]} \alpha_{-1}^{\mu} \alpha_{-2}^{\nu}+\epsilon_{\mu} \alpha_{-3}^{\mu}\right]|0, k\rangle} \\
& =\left|A\left(U_{C B A}\right)\right\rangle+\left|B\left(V_{\left[T_{i} A\right]}\right)\right\rangle+\left|C\left(U_{P B A}\right)\right\rangle \\
& +\sum_{A=L, T_{i}}\left[\frac{1}{9}\left(U_{L L A}+\sum_{i} U_{T_{i} T_{i} A}\right)\right]\left|D_{1}\left(e^{A}\right)\right\rangle \\
& -\frac{1}{9} \sum_{A=L, T_{i}} V_{[P A]}\left|D_{2}^{\prime}\left(e^{A}\right)\right\rangle \\
& +\frac{1}{75}\left[U_{L L P}+\sum_{i} U_{P T_{i} T_{i}}\right]|E\rangle .
\end{aligned}
$$

In Eq.(2.83), $\left|A\left(U_{C B A}\right)\right\rangle$ is given by Eq.(2.38) with $\tilde{u}_{C B A}$ given by Eq.(2.64) and we have replaced $u$ by $U$ on the r.h.s. of Eq.(2.64). The antisymmetric spin-two positive-norm state $\left|B\left(V_{\left[T_{i} A\right]}\right)\right\rangle$ is given by Eq.(2.42) and we have replaced $v$ by $V$ in Eq. (2.42). Finally, $\left|C\left(U_{P B A}\right)\right\rangle$ is given by Eq.(2.43) with $\theta$ given by Eq.(2.65) and we have replaced $u$ by $U$ on the r.h.s. of Eq.(2.65). In Eq.(2.85), $\left|D_{2}^{\prime}\left(e^{A}\right)\right\rangle \equiv\left|D_{2}\left(e^{A}\right)\right\rangle-2\left|D_{1}\left(e^{A}\right)\right\rangle$ is the inter-particle zero-norm state introduced in the end of subsection A with $\alpha=-2$. Note that the value of $\alpha$ is a choice of convension fixed by the parametrizations of the polarizations. It can always be adjusted to be zero. In view of Eqs.(2.77) and (2.78), we see that one can use either $V_{[P A]}$ or $U_{P P A}^{(2)}\left(A=L, T_{i}\right)$ to represent the polarization of the $\left|D_{2}^{\prime}\left(e^{A}\right)\right\rangle$ inter-particle zero-norm state.

We conclude that once we turn on the antisymmetric spin-two positive-norm state in the general representation of physical states Eq.(2.66), it is naturally accompanied by the $D_{2}^{\prime}$ inter-particle zero-norm state. The polarization of the $D_{2}^{\prime}$ inter-particle zero-norm state 
can be represented by either $V_{[P A]}$ or $U_{P P A}^{(2)}\left(A=L, T_{i}\right)$ in Eqs.(2.72) and (2.73). Thus this inter-particle zero-norm state will generate an inter-particle symmetry transformation in the $\sigma$-model calculation considered in $[7,13]$. Note that, in contrast to the high-energy symmetry of Gross [5], this symmetry is valid to all orders in $\alpha^{\prime}$.

\section{LIGHT-CONE DDF ZERO-NORM STATES}

In the usual light-cone quantization of bosonic string theory, one solves the Virasoro constraints to get rid of two string coordinates $X^{ \pm}$. Only 24 string coordinates $\alpha_{n}^{i}, i=$ $1, \ldots, 24$, remain, and there are no zero-norm states in the spectrum. However, there exists another related quantization scheme, the DDF quantization, which does include the zeronorm states in the spectrum. In the light-cone DDF quantization of open bosonic string [17], one constructs transverse physical states with discrete momenta

$$
p^{\mu}=p_{0}^{\mu}-N k_{0}^{\mu}=(1,0 \ldots .,-1+N),
$$

where $X^{ \pm} \equiv \frac{1}{\sqrt{2}}\left(X^{0} \pm X^{25}\right)$ and $p^{+}=1, p^{-}=-1+N$. In Eq.(3.1) $m^{2}=-p^{2}=2(N-1)$ and $p_{0}^{\mu} \equiv(1,0 \ldots,-1), k_{0}^{\mu} \equiv(0,0 \ldots,-1)$, respectively. All other momenta can be reached by Lorentz transformations. The DDF operators are given by [17]

$$
A_{n}^{i}=\frac{1}{2 \pi} \int_{0}^{2 \pi} \dot{X}^{i}(\tau) e^{i n X^{+}(\tau)} d \tau, i=1, \ldots,, 24
$$

where the massless vertex operator $V^{i}\left(n k_{0}, \tau\right)=\dot{X}^{i}(\tau) e^{i n X^{+}(\tau)}$ is a primary field with conformal dimension one, and is periodic in the worldsheet time $\tau$ if one chooses $k^{\mu}=n k_{0}^{\mu}$ with $n \in Z$. It is then easy to show that

$$
\begin{aligned}
& {\left[L_{m}, A_{n}^{i}\right]=0} \\
& {\left[A_{m}^{i}, A_{n}^{j}\right]=m \delta_{i j} \delta_{m+n} .}
\end{aligned}
$$

In addition to sharing the same algebra, Eq.(3.4), with string coordinates $\alpha_{n}^{i}$, the DDF operators $A_{n}^{i}$ possess a nicer property Eq.(3.3), which enables us to easily write down a general formula for the positive-norm physical states as following

$$
\left(A_{-1}^{j}\right)^{i_{1}}\left(A_{-2}^{k}\right)^{i_{2}} \ldots\left(A_{-m}^{l}\right)^{i_{m}} \mid 0, p_{0}>, i_{r} \in N
$$


where $\mid 0, p_{0}>$ is the tachyon ground state and $N=\sum_{r=1}^{m} r i_{r}$ is the level of the state. Historically, DDF operators were used to prove no-ghost (negative-norm states) theorem for $D=26$ string theory. Here we are going to use them to analyse zero-norm states. It turns out that zero-norm states can be generated by

$$
\tilde{A}_{n}^{-}=A_{n}^{-}-\sum_{m=1}^{\infty} \sum_{i=1}^{D-2}: A_{m}^{i} A_{n-m}^{i}:
$$

where $A_{n}^{-}$is given by

$$
A_{n}^{-}=\frac{1}{2 \pi} \int_{0}^{2 \pi}\left[: \dot{X}^{-} e^{i n X^{+}}:-\frac{1}{2} i n \frac{d}{d \tau}\left(\log \dot{X}^{+}\right) e^{i n X^{+}}\right] d \tau .
$$

It can be shown that $\tilde{A}_{n}^{-}$commute with $L_{m}$ and satisfy the following algebra

$$
\begin{aligned}
& {\left[\tilde{A}_{m}^{-}, A_{n}^{i}\right]=0} \\
& {\left[\tilde{A}_{m}^{-}, \tilde{A}_{n}^{-}\right]=(m-n) \tilde{A}_{m+n}^{-}+\frac{26-D}{12} m^{3} \delta_{m+n}}
\end{aligned}
$$

Eqs.(3.4), (3.8) and (3.9) constitute the spectrum generating algebra for the open bosonic string including zero-norm states. The ground state $\left|0, p_{0}>\equiv\right| 0>$ satisfies the following conditions

$$
\begin{aligned}
& A_{n}^{i}\left|0>=\tilde{A}_{n}^{-}\right| 0>=0, \quad n>0, \\
& \tilde{A}_{0}^{-}\left|0>=-\frac{26-D}{24}, \quad A_{0}^{i}\right| 0>=0 .
\end{aligned}
$$

We are now ready to construct zero-norm states in the DDF formalism.

1. $m^{2}=0$ : One has only one scalar $\tilde{A}_{-1}^{-} \mid 0>$, which has zero-norm for any $D$.

2. $m^{2}=2$ : One has a light-cone vector $A_{-1}^{i} \tilde{A}_{-1}^{-} \mid 0>$, which has zero-norm for any $D$, and two scalars, whose norms are calculated to be

$$
\left\|\left(a \tilde{A}_{-1}^{-} \tilde{A}_{-1}^{-}+b \tilde{A}_{-2}^{-}\right) \mid 0>\right\|=\frac{26-D}{2} b^{2} .
$$

For $b=0$, one has a "pure type I" zero-norm state, $\tilde{A}_{-1}^{-} \tilde{A}_{-1}^{-} \mid 0>$, which has zero-norm for any $D$. By combining with the light-cone vector $A_{-1}^{i} \tilde{A}_{-1}^{-} \mid 0>$, one obtains a vector zeronorm state with 25 degrees of freedom, which correspond to Eq.(2.5) in the OCFQ approach. For $b \neq 0$, one obtains a type II scalar zero-norm state for $D=26$, which corresponds to Eq.(2.4) in the OCFQ approach.

3. $m^{2}=4$ : 
I. A spin-two tensor $A_{-1}^{i} A_{-1}^{j} \tilde{A}_{-1}^{-} \mid 0>$, which has zero-norm for any $D$.

II. Three light-cone vectors, whose norms are calculated to be

$$
\left\|\left(a A_{-1}^{i} \tilde{A}_{-1}^{-} \tilde{A}_{-1}^{-}+b A_{-2}^{i} \tilde{A}_{-1}^{-}+c A_{-1}^{i} \tilde{A}_{-2}^{-}\right) \mid 0>\right\|=\frac{26-D}{2} c^{2} .
$$

III. Three scalars, whose norms are calculated to be

$$
\left\|\left(d \tilde{A}_{-1}^{-} \tilde{A}_{-1}^{-} \tilde{A}_{-1}^{-}+e \tilde{A}_{-1}^{-} \tilde{A}_{-2}^{-}+f \tilde{A}_{-3}^{-}\right) \mid 0>\right\|=2(26-D)(e+f)^{2} .
$$

For $c=0$ in Eq.(3.13), one has two "pure type I" light-cone vector zero-norm states. For $e+f=0$ in Eq.(3.14), one has two "pure type I" scalar zero-norm states. One of the two type I light-cone vectors, when combining with the spin-two state in I, gives the type I spin-two tensor which corresponds to Eq.(2.7) in the OCFQ approach. The other type I light-cone vector, when combining with one of the two type I scalar, gives the type I vector zero-norm state which corresponds to Eq.(2.8) in the OCFQ approach. The other type I scalar corresponds to Eq.(2.9). Finally, for $c \neq 0$ and $e+f \neq 0$, one obtains the type II vector zero-norm state for $D=26$, which corresponds to Eq.(2.6) in the OCFQ approach. It is easy to see that a special linear combination of $b$ and $c$ will give the inter-particle vector zero-norm state which corresponds to the inter-particle $D_{2}$ zero-norm state in Eq (2.10). This completes the analysis of zero-norm states for $m^{2}=4$. Note that the exact mapping of zero-norm states in the light-cone DDF formalism and the OCFQ approach depends on the exact relation between operators $\left(\tilde{A}_{n}^{-}, A_{n}^{i}, L_{n}\right)$ and $\alpha_{n}^{\mu}$, which has not been worked out in the literature.

\section{BRST ZERO-NORM STATES IN WSFT}

Cubic string field theory is defined on a disk with the action

$$
S=-\frac{1}{g_{0}}\left(\frac{1}{2} \int \Phi * Q_{\mathrm{B}} \Phi+\frac{1}{3} \int \Phi * \Phi * \Phi\right),
$$

where $Q_{\mathrm{B}}$ is the BRST charge

$$
Q_{\mathrm{B}}=\sum_{n=-\infty}^{\infty} L_{-n}^{\mathrm{m}} c_{n}+\sum_{m, n=-\infty}^{\infty} \frac{m-n}{2}: c_{m} c_{n} b_{-m-n}:-c_{0}
$$


and $\Phi$ is the string field with ghost number 1 and $b, c$ are conformal ghosts. Since the ghost number of vacuum on a disk is -3 , the total ghost number of this action is 0 as expected. The string field can be expanded as

$$
\Phi=\sum_{k, m, n} A_{\mu \cdots, k \cdots m \cdots n \cdots}(x) \alpha_{k}^{\mu} \cdots b_{m} \cdots c_{n} \cdots|\Omega\rangle
$$

where the string ground state $|\Omega\rangle$ is

$$
|\Omega\rangle=c_{1}|0\rangle
$$

The gauge transformation for string field can be written as

$$
\delta \Phi=Q_{\mathrm{B}} \Lambda+g(\Phi * \Lambda-\Lambda * \Phi)
$$

where $\Lambda$ is the a string field with ghost number 0 .

For the purpose of discussion in this paper, we are going to consider the linearized gauge transformation

$$
\delta \Phi=Q_{\mathrm{B}} \Lambda
$$

where $Q_{\mathrm{B}} \Lambda$ is just the off-shell zero-norm states. In the following, we will explicitly show that the components of Eq.(4.5) are in one-to-one correspondence to the zero-norm states obtained in OCFQ approach in section II level by level for the first few mass levels.

There is no zero-norm state in the lowest string mass level with $m^{2}=-2$, so our analysis will start with the mass level of $m^{2}=0$.

$\underline{m^{2}=0:}$

The string field can be expanded as

$$
\begin{aligned}
& \Phi=\left\{i A_{\mu}(x) \alpha_{-1}^{\mu}+\alpha(x) b_{-1} c_{0}\right\}|\Omega\rangle, \\
& \Lambda=\left\{\epsilon^{0}(x) b_{-1}\right\}|\Omega\rangle .
\end{aligned}
$$

The gauge transformation is then

$$
Q_{\mathrm{B}} \Lambda=\left\{-\frac{1}{2} \alpha_{0}^{2} \epsilon^{0} b_{-1} c_{0}+\epsilon^{0} \alpha_{0} \cdot \alpha_{-1}\right\}|\Omega\rangle .
$$

The nilpotency of BRST charge $Q_{\mathrm{B}}$ gives

$$
Q_{\mathrm{B}}^{2} \Lambda=0,
$$


which can be easily checked to be valid for any D. Thus Eq.(4.8) can be interpreted as a type I zero-norm state. To compare it with the zero-norm state obtained in OCFQ approach in section II, we need to reduce the Hilbert space by removing the ghosts states. In particular, the coefficients of terms with ghost operaters must vanish. For the state in Eq.(4.8), it is

$$
\alpha_{0}^{2} \epsilon^{0}=0
$$

which give the on-shell condition $k^{2}=0$ and the following zero-norm state

$$
Q_{\mathrm{B}} \Lambda=\epsilon^{0} \alpha_{0} \cdot \alpha_{-1}|\Omega\rangle
$$

This is the same as the scalar zero-norm state obtained in OCFQ approach.

$\underline{m^{2}=2:}$

The string fields expansion are

$$
\begin{aligned}
\Phi=\{ & -B_{\mu \nu}(x) \alpha_{-1}^{\mu} \alpha_{-1}^{\nu}+i B_{\mu}(x) \alpha_{-2}^{\mu} \\
& \left.\quad+i \beta_{\mu}(x) \alpha_{-1}^{\mu} b_{-1} c_{0}+\beta^{0}(x) b_{-2} c_{0}+\beta^{1}(x) b_{-1} c_{-1}\right\}|\Omega\rangle, \\
\Lambda=\{ & \left.i \epsilon_{\mu}^{0}(x) \alpha_{-1}^{\mu} b_{-1}+\epsilon^{1}(x) b_{-2}\right\}|\Omega\rangle .
\end{aligned}
$$

The off-shell zero-norm states are calculated to be

$$
\begin{aligned}
Q_{\mathrm{B}} \Lambda=\{ & \left(i \alpha_{0 \mu} \epsilon_{\nu}^{0}+\frac{1}{2} \epsilon^{1} \eta_{\mu \nu}\right) \alpha_{-1}^{\mu} \alpha_{-1}^{\nu}+\left(i \epsilon^{0}+\alpha_{0} \epsilon^{1}\right) \cdot \alpha_{-2} \\
& -i \frac{1}{2}\left(\alpha_{0}^{2}+2\right)\left(\epsilon^{0} \cdot \alpha_{-1}\right) b_{-1} c_{0}-\frac{1}{2}\left(\alpha_{0}^{2}+2\right) \epsilon^{1} b_{-2} c_{0} \\
& \left.-\left(i \alpha_{0} \cdot \epsilon^{0}+3 \epsilon^{1}\right) b_{-1} c_{-1}\right\}|\Omega\rangle .
\end{aligned}
$$

Nilpotency condition requires

$$
Q_{\mathrm{B}}^{2} \Lambda=\frac{D-26}{2} \epsilon^{1} c_{-2}|\Omega\rangle=0
$$

There are two solutions of Eq.(4.15), which correspond to the type I and type II zero-norm states, respectively.

1. Type I: in this case $D$ is not restricted to the critical string dimension in Eq.(4.15), i.e. $D \neq 26$. Thus

$$
\epsilon^{1}=0
$$


The no-ghost conditions of Eq.(4.14) lead to the on-shell constraints

$$
\begin{aligned}
& \alpha_{0}^{2}+2=0, \\
& \alpha_{0} \cdot \epsilon^{0}=0 .
\end{aligned}
$$

The off-shell zero-norm state in Eq.(4.14) then reduces to an on-shell vector zero-norm state

$$
Q_{\mathrm{B}} \Lambda=i\left\{\left(\epsilon^{0} \cdot \alpha_{-1}\right)\left(\alpha_{0} \cdot \alpha_{-1}\right)+\epsilon^{0} \cdot \alpha_{-2}\right\}|\Omega\rangle
$$

2. Type II: in this case $D$ is restricted to the critical string dimension, i.e. $D=26$, and $\epsilon^{1}$ can be arbitrary function. The no-ghost conditions then lead to the on-shell constraints

$$
\begin{aligned}
\alpha_{0}^{2}+2 & =0, \\
i \alpha_{0} \cdot \epsilon^{0}+3 \epsilon^{1} & =0 .
\end{aligned}
$$

The second condition can be solved by a special solution

$$
\epsilon_{\mu}^{0}=-\frac{3 i}{2} \alpha_{0 \mu} \epsilon^{1}
$$

which leads to an on-shell scalar zero-norm state

$$
Q_{\mathrm{B}} \Lambda=\left\{\frac{3}{2}\left(\alpha_{0} \cdot \alpha_{-1}\right)^{2}+\frac{1}{2}\left(\alpha_{-1} \cdot \alpha_{-1}\right)+\frac{5}{2}\left(\alpha_{0} \cdot \alpha_{-2}\right)\right\} \epsilon^{1}|\Omega\rangle
$$

Again, up to a constant factor, the zero-norm states Eqs.(4.19) and (4.23) are the same as Eqs. (2.5) and (2.4) calculated in the OCFQ approach.

$\underline{m^{2}=4:}$

The string fields are expanded as

$$
\begin{aligned}
\Phi=\{ & -i C_{\mu \nu \lambda}(x) \alpha_{-1}^{\mu} \alpha_{-1}^{\nu} \alpha_{-1}^{\lambda}-C_{\mu \nu}(x) \alpha_{-2}^{\mu} \alpha_{-1}^{\nu}+i C_{\mu}(x) \alpha_{-3}^{\mu} \\
& \quad-\gamma_{\mu \nu}(x) \alpha_{-1}^{\mu} \alpha_{-1}^{\nu} b_{-1} c_{0}+i \gamma_{\mu}^{0}(x) \alpha_{-1}^{\mu} b_{-2} c_{0}+i \gamma_{\mu}^{1}(x) \alpha_{-1}^{\mu} b_{-1} c_{-1} \\
& \left.+i \gamma_{\mu}^{2}(x) \alpha_{-2}^{\mu} b_{-1} c_{0}+\gamma^{0}(x) b_{-3} c_{0}+\gamma^{1}(x) b_{-2} c_{-1}+\gamma^{2}(x) b_{-1} c_{-2}\right\}|\Omega\rangle, \\
\Lambda=\{ & -\epsilon_{\mu \nu}(x) \alpha_{-1}^{\mu} \alpha_{-1}^{\nu} b_{-1}+i \epsilon_{\mu}^{1}(x) \alpha_{-2}^{\mu} b_{-1}|\Omega\rangle \\
& \left.+i \epsilon_{\mu}^{2}(x) \alpha_{-1}^{\mu} b_{-2}+\epsilon^{2}(x) b_{-3}+\epsilon^{3}(x) b_{-1} b_{-2} c_{0}\right\}|\Omega\rangle .
\end{aligned}
$$


The off-shell zero-norm states are

$$
\begin{aligned}
Q_{\mathrm{B}} \Lambda=\{ & \left(-\alpha_{0(\mu} \epsilon_{\nu \lambda)}+\frac{i}{2} \epsilon_{(\mu}^{2} \eta_{\nu \lambda)}\right) \alpha_{-1}^{\mu} \alpha_{-1}^{\nu} \alpha_{-1}^{\lambda}+\left(i \alpha_{0 \mu} \epsilon_{\nu}^{2}+i \alpha_{0 \nu} \epsilon_{\mu}^{1}-2 \epsilon_{\mu \nu}+\epsilon^{2} \eta_{\mu \nu}\right) \alpha_{-2}^{\mu} \alpha_{-1}^{\nu} \\
& +\left(\alpha_{0 \mu} \epsilon^{2}+2 i \epsilon_{\mu}^{1}+i \epsilon_{\mu}^{2}\right) \alpha_{-3}^{\mu}+\left[\frac{1}{2}\left(\alpha_{0}^{2}+4\right) \epsilon_{\mu \nu}+\frac{1}{2} \epsilon^{3} \eta_{\mu \nu}\right] \alpha_{-1}^{\mu} \alpha_{-1}^{\nu} b_{-1} c_{0} \\
& +\left[-\frac{i}{2}\left(\alpha_{0}^{2}+4\right) \epsilon_{\mu}^{2}-\alpha_{0 \mu} \epsilon^{3}\right] \alpha_{-1}^{\mu} b_{-2} c_{0}+\left(2 \alpha_{0}^{\nu} \epsilon_{\nu \mu}-2 i \epsilon_{\mu}^{1}-3 i \epsilon_{\mu}^{2}\right) \alpha_{-1}^{\mu} b_{-1} c_{-1} \\
& +\left[-\frac{i}{2}\left(\alpha_{0}^{2}+4\right) \epsilon_{\mu}^{1}+\alpha_{0 \mu} \epsilon^{3}\right] \alpha_{-2}^{\mu} b_{-1} c_{0}+\left[-\frac{1}{2}\left(\alpha_{0}^{2}+4\right) \epsilon^{2}-\epsilon^{3}\right] b_{-3} c_{0} \\
& \left.+\left(-i \alpha_{0}^{\mu} \epsilon_{\mu}^{2}-4 \epsilon^{2}-2 \epsilon^{3}\right) b_{-2} c_{-1}+\left(-2 i \alpha_{0}^{\mu} \epsilon_{\mu}^{1}-5 \epsilon^{2}+4 \epsilon^{3}+\epsilon_{\mu}^{\mu}\right) b_{-1} c_{-2}\right\}|\Omega\rangle .
\end{aligned}
$$

Nilpotency condition requires

$$
Q_{\mathrm{B}}^{2} \Lambda=(D-26)\left[\frac{i}{2} \epsilon_{\mu}^{2} \alpha_{-1}^{\mu} c_{-2}+2 \epsilon^{2} c_{-3}-\frac{1}{2} \epsilon^{3} b_{-1} c_{-2} c_{0}\right]=0 .
$$

Similarly, we classify the solutions of Eq. (4.27) by type I and type II in the following:

1. Type I: $D \neq 26$. This leads to

$$
\epsilon^{2}=\epsilon^{3}=\epsilon_{\mu}^{2}=0
$$

The no-ghost conditions lead to the on-shell constraints

$$
\begin{aligned}
\alpha_{0}^{2}+4 & =0, \\
\alpha_{0}^{\nu} \epsilon_{\nu \mu}-i \epsilon_{\mu}^{1} & =0, \\
-2 i\left(\alpha_{0} \cdot \epsilon^{1}\right)+\epsilon_{\mu}^{\mu} & =0 .
\end{aligned}
$$

One can apply the same technique as in subsection IIB to obtain a complete set of solutions to Eqs.(4.30) and (4.31). However, for simplicity, we shall list all independent solutions only. There are three independent solutions to the above equations, which correspond to the three type I on-shell zero-norm states:

- Tensor zero-norm state

$$
\begin{gathered}
\epsilon_{\mu}^{1}=0, \quad \alpha_{0}^{\nu} \epsilon_{\mu \nu}=0, \quad \epsilon_{\mu}^{\mu}=0 \\
Q_{\mathrm{B}} \Lambda=-\left\{\alpha_{0 \mu} \epsilon_{\nu \lambda} \alpha_{-1}^{\mu} \alpha_{-1}^{\nu} \alpha_{-1}^{\lambda}+2 \epsilon_{\mu \nu} \alpha_{-2}^{\mu} \alpha_{-1}^{\nu}\right\}|\Omega\rangle .
\end{gathered}
$$


- Vector zero-norm state

$$
\begin{gathered}
\alpha_{0} \cdot \epsilon^{1}=0, \quad \epsilon_{\mu \nu}=-\frac{i}{4}\left(\alpha_{0 \nu} \epsilon_{\mu}^{1}+\alpha_{0 \mu} \epsilon_{\nu}^{1}\right), \\
Q_{\mathrm{B}} \Lambda=\left\{i \frac{1}{2}\left(\alpha_{0} \cdot \alpha_{-1}\right)^{2}\left(\epsilon^{1} \cdot \alpha_{-1}\right)+2 i\left(\epsilon^{1} \cdot \alpha_{-3}\right)\right. \\
\left.+\frac{3}{2}\left(\alpha_{0} \cdot \alpha_{-1}\right)\left(\epsilon^{1} \cdot \alpha_{-2}\right)+\frac{1}{2}\left(\alpha_{0} \cdot \alpha_{-2}\right)\left(\epsilon^{1} \cdot \alpha_{-1}\right)\right\}|\Omega\rangle .
\end{gathered}
$$

- Scalar zero-norm state

$$
\begin{gathered}
\epsilon_{\mu}^{1}=\frac{i(D-1)}{9} \alpha_{0 \mu} \theta, \quad \epsilon_{\mu \nu}=\eta_{\mu \nu} \theta+\frac{(8+D)}{36} \alpha_{0 \mu} \alpha_{0 \nu} \theta \\
Q_{\mathrm{B}} \Lambda=-\frac{2}{9}\left\{\frac{(8+D)}{8}\left(\alpha_{0} \cdot \alpha_{-1}\right)^{3}+\frac{9}{2}\left(\alpha_{0} \cdot \alpha_{-1}\right)\left(\alpha_{-1} \cdot \alpha_{-1}\right)+9\left(\alpha_{-1} \cdot \alpha_{-2}\right)\right. \\
\left.+\frac{3(D+2)}{4}\left(\alpha_{0} \cdot \alpha_{-1}\right)\left(\alpha_{0} \cdot \alpha_{-2}\right)+(D-1)\left(\alpha_{0} \cdot \alpha_{-3}\right)\right\} \theta|\Omega\rangle
\end{gathered}
$$

If we set $D=26$, then

$$
\begin{aligned}
Q_{\mathrm{B}} \Lambda= & -\frac{2}{9}\left\{\frac{17}{4}\left(\alpha_{0} \cdot \alpha_{-1}\right)^{3}+\frac{9}{2}\left(\alpha_{0} \cdot \alpha_{-1}\right)\left(\alpha_{-1} \cdot \alpha_{-1}\right)+9\left(\alpha_{-1} \cdot \alpha_{-2}\right)\right. \\
& \left.+21\left(\alpha_{0} \cdot \alpha_{-1}\right)\left(\alpha_{0} \cdot \alpha_{-2}\right)+25\left(\alpha_{0} \cdot \alpha_{-3}\right)\right\} \theta|\Omega\rangle,
\end{aligned}
$$

where $\theta$ is an arbitrary function.

2. Type II: $D=26$ in Eq.(4.27), and $\epsilon^{2}, \epsilon^{3}$ and $\epsilon_{\mu}^{2}$ are arbitrary functions. The no-ghost conditions lead to the on-shell constraints

$$
\begin{aligned}
\alpha_{0}^{2}+4 & =0, \\
\epsilon^{3} & =0, \\
2 \alpha_{0}^{\nu} \epsilon_{\nu \mu}-2 i \epsilon_{\mu}^{1}-3 i \epsilon_{\mu}^{2} & =0, \\
i \alpha_{0}^{\mu} \epsilon_{\mu}^{2}+4 \epsilon^{2} & =0, \\
-2 i \alpha_{0}^{\mu} \epsilon_{\mu}^{1}-5 \epsilon^{2}+\epsilon_{\mu}^{\mu} & =0 .
\end{aligned}
$$

In addition to all three type I zero-norm states as found above, we now have a new solution to the above equations. This special solution can be chosen as

$$
\begin{aligned}
\epsilon^{2} & =-\frac{i}{4}\left(\alpha_{0} \cdot \epsilon^{2}\right)=0, \\
\epsilon_{\mu \nu} & =-C\left(\alpha_{0 \mu} \epsilon_{\nu}^{2}+\alpha_{0 \nu} \epsilon_{\mu}^{2}\right), \\
\epsilon_{\mu}^{1} & =\frac{8 i C-3}{2} \epsilon_{\mu}^{2},
\end{aligned}
$$


which gives an on-shell vector zero-norm state

$$
\begin{aligned}
Q_{\mathrm{B}} \Lambda=i\{ & (8 i C-2)\left(\epsilon^{2} \cdot \alpha_{-3}\right)+\frac{1}{2}\left(\alpha_{-1} \cdot \alpha_{-1}\right)\left(\epsilon^{2} \cdot \alpha_{-1}\right) \\
& +(2 i C+1)\left(\alpha_{0} \cdot \alpha_{-2}\right)\left(\epsilon^{2} \cdot \alpha_{-1}\right)+2 i C\left(\alpha_{0} \cdot \alpha_{-1}\right)^{2}\left(\epsilon^{2} \cdot \alpha_{-1}\right) \\
& \left.+\frac{12 i C-3}{2}\left(\alpha_{0} \cdot \alpha_{-1}\right)\left(\epsilon^{2} \cdot \alpha_{-2}\right)\right\}|\Omega\rangle .
\end{aligned}
$$

For a special value of $C=-3 i / 4$, Eq.(4.47) becomes

$$
\begin{aligned}
Q_{\mathrm{B}} \Lambda=i\{ & 4\left(\epsilon^{2} \cdot \alpha_{-3}\right)+\frac{1}{2}\left(\alpha_{-1} \cdot \alpha_{-1}\right)\left(\epsilon^{2} \cdot \alpha_{-1}\right)+\frac{5}{2}\left(\alpha_{0} \cdot \alpha_{-2}\right)\left(\epsilon^{2} \cdot \alpha_{-1}\right) \\
& \left.+\frac{3}{2}\left(\alpha_{0} \cdot \alpha_{-1}\right)^{2}\left(\epsilon^{2} \cdot \alpha_{-1}\right)+3\left(\alpha_{0} \cdot \alpha_{-1}\right)\left(\epsilon^{2} \cdot \alpha_{-2}\right)\right\}|\Omega\rangle .
\end{aligned}
$$

Up to a constant factor, zero-norm states in Eqs.(4.33), (4.35), (4.38) and (4.48) are exactly the same as Eqs.( 2.7), (2.8), (2.9) and (2.6) calculated in the OCFQ approach. In addition, it can be checked that for $C=-5 i / 8$ and $-i / 16$ in Eq.(4.47), one gets $D_{1}$ and $D_{2}$ zero-norm states of OCFQ approach in Eqs.(11) and (10), respectively.

In Ref [16], the background ghost transformations in the gauge transformations of WSFT [15] were shown to correspond, in a one-to-one manner, to the lifting of on-shell conditions of zero-norm states in the OCFQ approach. For the rest of this section, we are going to go one step further and apply the results calculated above to demonstrate that off-shell gauge transformations of WSFT are indeed identical to the on-shell stringy gauge symmetries generated by two types of zero-norm states in the generalized massive $\sigma$-model approach [7] of string theory. For the mass level $m^{2}=2$, by using Eqs.(4.12) and (4.13), the linearized gauge transformation of WSFT in Eq.(4.5) gives

$$
\begin{aligned}
\delta B_{\mu \nu} & =-\partial_{(\mu} \epsilon_{\nu)}^{0}-\frac{1}{2} \epsilon^{1} \eta_{\mu \nu} \\
\delta B_{\mu} & =-\partial_{\mu} \epsilon^{1}+\frac{1}{2} \epsilon_{\mu}^{0} \\
\delta \beta_{\mu} & =\frac{1}{2}\left(\partial^{2}-2\right) \epsilon_{\mu}^{0} \\
\delta \beta^{0} & =\frac{1}{2}\left(\partial^{2}-2\right) \epsilon^{1} \\
\delta \beta^{1} & =-\partial^{\mu} \epsilon_{\mu}^{0}-3 \epsilon^{1}
\end{aligned}
$$

For the type I gauge transformation induced by zero-norm state in Eq.(2.5), one can use Eqs.(4.16) -(4.18) to eliminate the background ghost transformations Eqs.(4.51)- 
(4.53). Finally, conditions of worldsheet conformal invariance in the presence of weak background fields [7] can be used to express $B_{\mu}$ in terms of $B_{\mu \nu}$, and one ends up with the following on-shell gauge transformation by Eq.(4.49)

$$
\delta B_{\mu \nu}=\partial_{(\mu} \epsilon_{\nu)}^{0} ; \quad \partial^{\mu} \epsilon_{\mu}^{0}=0, \quad\left(\partial^{2}-2\right) \epsilon_{\mu}^{0}=0 .
$$

Similarly, one can apply the same procedure to type II zero-norm state in Eq.(2.4), and derive the following type II gauge transformation

$$
\delta B_{\mu \nu}=\frac{3}{2} \partial_{\mu} \partial_{\nu} \epsilon^{1}-\frac{1}{2} \eta_{\mu \nu} \epsilon^{1}, \quad\left(\partial^{2}-2\right) \epsilon^{1}=0 .
$$

Eqs.(4.54) and (4.55) are consistent with the massive $\sigma$-model calculation in the OCFQ string theory in [7].

For the mass level $m^{2}=4$, by using Eqs.(4.24) and (4.25), the linearized gauge transformation of WSFT in Eq.(4.5) gives

$$
\begin{aligned}
\delta C_{\mu \nu \lambda} & =-\partial_{(\mu} \epsilon_{\nu \lambda)}^{0}-\frac{1}{2} \epsilon_{(\mu}^{2} \eta_{\mu \nu)}, \\
\delta C_{[\mu \nu]} & =-\partial_{[\nu} \epsilon_{\mu]}^{1}-\partial_{[\mu} \epsilon_{\nu]}^{2}, \\
\delta C_{(\mu \nu)} & =-\partial_{(\nu} \epsilon_{\mu)}^{1}-\partial_{(\mu} \epsilon_{\nu)}^{2}+2 \epsilon_{\mu \nu}^{0}-\epsilon^{2} \eta_{\mu \nu}, \\
\delta C_{\mu} & =-\partial_{\mu} \epsilon^{2}+2 \epsilon_{\mu}^{1}+\epsilon_{\mu}^{2}, \\
\delta \gamma_{\mu \nu} & =\frac{1}{2}\left(\partial^{2}-4\right) \epsilon_{\mu \nu}^{0}-\frac{1}{2} \epsilon^{3} \eta_{\mu \nu}, \\
\delta \gamma_{\mu}^{0} & =\frac{1}{2}\left(\partial^{2}-4\right) \epsilon_{\mu}^{2}+\partial_{\mu} \epsilon^{3}, \\
\delta \gamma_{\mu}^{1} & =-2 \partial^{\nu} \epsilon_{\nu \mu}^{0}-2 \epsilon_{\mu}^{1}-3 \epsilon_{\mu}^{2}, \\
\delta \gamma_{\mu}^{2} & =\frac{1}{2}\left(\partial^{2}-4\right) \epsilon_{\mu}^{1}-\partial_{\mu} \epsilon^{3}, \\
\delta \gamma^{0} & =\frac{1}{2}\left(\partial^{2}-4\right) \epsilon^{2}-\epsilon^{3}, \\
\delta \gamma^{1} & =-\partial^{\mu} \epsilon_{\mu}^{2}-4 \epsilon^{2}-2 \epsilon^{3}, \\
\delta \gamma^{2} & =-2 \partial^{\mu} \epsilon_{\mu}^{1}-5 \epsilon^{2}+4 \epsilon^{3}+\epsilon_{\mu}^{0 \mu} .
\end{aligned}
$$

For the gauge transformation induced by $D_{2}$ zero-norm state in Eq.(4.48), for example, one can use Eqs.(4.39)-(4.46) with $C=-i / 16$ to eliminate Eqs.(4.60)-(4.66). One can then use the fact that background fields $C_{(\mu \nu)}$ and $C_{\mu}$ are gauge artifacts of $C_{\mu \nu \lambda}$ in the $\sigma$-model calculation, and deduce from Eqs.(4.56)-(4.59) the inter-particle symmetry transformation

$$
\delta C_{\mu \nu \lambda}=\frac{1}{2} \partial_{(\mu} \partial_{\nu} \epsilon_{\lambda)}^{\left(D_{2}\right)}-2 \eta_{(\mu \nu} \epsilon_{\lambda)}^{\left(D_{2}\right)}, \quad \delta C_{[\mu \nu]}=9 \partial_{[\mu} \epsilon_{\nu]}^{\left(D_{2}\right)},
$$


where $\partial^{\lambda} \epsilon_{\lambda}^{\left(D_{2}\right)}=0,\left(\partial^{2}-4\right) \epsilon_{\lambda}^{\left(D_{2}\right)}=0$. The other three gauge transformations corresponding to three other zero-norm states, the spin-two, $D_{1}$, and scalar can be similarly constructed from Eqs.(4.56)-(4.66). One gets

$$
\begin{gathered}
\delta C_{\mu \nu \lambda}=\partial_{(\mu} \epsilon_{\nu \lambda)} ; \quad \partial^{\mu} \epsilon_{\mu \nu}=0,\left(\partial^{2}-4\right) \epsilon_{\mu \nu}=0 \\
\delta C_{\mu \nu \lambda}=\frac{5}{2} \partial_{(\mu} \partial_{\nu} \epsilon_{\lambda)}^{\left(D_{1}\right)}-\eta_{(\mu \nu} \epsilon_{\lambda)}^{\left(D_{1}\right)} ; \partial^{\lambda} \epsilon_{\lambda}^{\left(D_{1}\right)}=0,\left(\partial^{2}-4\right) \epsilon_{\lambda}^{\left(D_{1}\right)}=0, \\
\delta C_{\mu \nu \lambda}=\frac{17}{4} \partial_{\mu} \partial_{\nu} \partial_{\lambda} \theta-\frac{9}{2} \eta_{(\mu \nu} \theta_{\lambda)} ;\left(\partial^{2}-4\right) \theta=0 .
\end{gathered}
$$

Eqs.(4.67)-(4.70) are exactly the same as those calculated by the generalized massive $\sigma$-model approach of string theory [7].

We thus have shown in this section that off-shell gauge transformations of WSFT are identical to the on-shell stringy gauge symmetries generated by two types of zero-norm states in the OCFQ string theory. The high energy limit of these stringy gauge symmetries generated by zero-norm states was recently used to calculate the proportionality constants among high energy scattering amplitudes of different string states conjectured by Gross [5]. Based on the zero-norm state calculations in [1-3] and the calculations in this section, we thus have related gauge symmetry of WSFT [15] to the high-energy stringy symmetry conjectured by Gross [4-6].

\section{CONCLUSION}

In this paper, we have calculated zero-norm states in the OCFQ string theory, the lightcone DDF string theory and the off-shell BRST string theory. In the OCFQ string theory, we have solved the Virasoro constraints for all physical states (including zero-norm states) in the helicity basis. Much attention is paid to discuss the inter-particle zero-norm state at the mass level $m^{2}=4$. We found that one can use polarization of either one of the two positive-norm states to represent the polarization of the inter-particle zero-norm state. This justifies how one can have the inter-particle symmetry transformation for the two massive modes in the weak field massive $\sigma$-model calculation derived previously [7]. This interparticle symmetry transformation, in contrast to the high energy symmetry of Gross [5], is valid to all energy.

In the light-cone DDF string theory, one can easily write down the general formula for all zero-norm states in the spectrum. We have identified type I and Type II zero-norm states 
up to the mass level $m^{2}=4$. The analysis can be easily generalized to any higher mass level as well.

Finally, we have calculated off-shell zero-norm states in the WSFT. After imposing the no ghost conditions, we can recover two types of on-shell zero-norm states in the OCFQ string theory. We then show that off-shell gauge transformations of WSFT are identical to the on-shell stringy gauge symmetries generated by two typse of zero-norm states in the generalized massive $\sigma$-model approach of string theory. Based on these zero-norm state calculations, we have thus related gauge symmetry of WSFT [15] to the high-energy stringy symmetry of Gross [5].

[1] C.T.Chan and J.C.Lee, "Stringy symmetries and their high energy limits", hep-th/0312226.

[2] C.T.Chan and J.C.Lee, Nucl.Phys. B690 3 (2004).

[3] C.T.Chan, P.M.Ho and J.C.Lee, "Ward identities and high energy scattering amplitudes in string theory", hep-th/0410194. (Accepted by Nucl.Phys. B)

[4] D.J.Gross and P. Mende, Phys. Lett. B197,129 (1987); Nucl.Phys.B303, 407(1988).

[5] D.J.Gross, "High energy symmetry of string theory", Phys. Rev. Lett. 60,1229 (1988); Phil.Trans. R. Soc. Lond. A329,401(1989).

[6] D.J.Gross and J.L. Manes, "The high energy behavior of open string theory", Nucl.Phys.B326, 73 (1989). See section 6 for details.

[7] J.C.Lee, Phys. Lett. B241, 336 (1990); J.C. Lee, Phys. Rev. Lett. 64, 1636 (1990). J.C. Lee and B.Ovrut, Nucl. Phys. B336, 222 (1990).

[8] J.C.Lee, Prog. of Theor. Phys. Vol. 91, 353 (1994), Phys. Lett. B, 33769 (1994).

[9] T.D.Chung and J.C.Lee, Phys. Lett. B350, 22 (1995); Z. Phys. C75, 555(1997). J.C. Lee, Eur.Phys.C, 739 (1998).

[10] J. Avan and A.Jevicki, Phys. Lett. B266, 35 (1991); B272, 17 (1991). I.R. Klebanov and A.M. Polyakov, Mod. Phys. Lett. A6, 3273 (1991).

[11] For a review see I.R. Klebanov and A. Pasquinucci, hep-th/9210105 and references therein.

[12] E. Witten, Nucl. Phys. B373, 187 (1992); E.Witten and B. Zwiebach, Nucl. Phys. B377, 55 (1992).

[13] J.C. Lee,"Calculations of zero-norm states and reduction of stringy scattering amplitudes" 
hep-th/0302123.

[14] J.L.Manes and M.A.H.Vozmediano, Nucl. Phys. B326, 271(1989).

[15] E. Witten, Nucl. Phys. B268, 253 (1986).

[16] H.C.Kao and J.C.Lee, Phys. Rev. D67,086003 (2003).

[17] E. Del Giudice, P. Di Vecchia and S.Fubine, Ann. of Phys.70, 378(1972), R.C.Brower and P.Goddard, Nucl. Phys. B40, 437 (1972), R.C. Brower, Phys. Rev. D6, 1655 (1972). Here we follow the discussion of Ref [18].

[18] M.Green, J.H.Schwarz and E.Witten, "Superstring Theory" Vol. I Cambridge University Press. 